\title{
Relações de atributos do solo e estabilidade de agregados em canaviais com e sem vinhaça ${ }^{1}$
}

\author{
Thais F. da S. Vicente', Elvira M. R. Pedrosa ${ }^{2}$, Mario M. Rolim², \\ Veronildo S. O liveira', Ana Karina S. O liveira ${ }^{2} \&$ Anailda M. P. L. Souza ${ }^{3}$
}

RESU M O

Realizou-se este estudo com o objetivo de se avaliar o efeito da aplicação da vinhaça na estabilidade dos agregados e nos índices de agregação do solo e nas correlações das propriedades físicas do solo em áreas cultivadas com cana-de-açúcar no litoral sul do estado de Pernambuco. As amostras de solo foram coletadas nas camadas de 0-0,20 e 0,20-0,40 m, em 12 áreas com e sem aplicação de vinhaça. Análises foram realizadas para caracterização do solo e também para determinação da argila dispersa em água, grau de floculação das argilas, carbono orgânico total e matéria orgânica do solo. A estabilidade dos agregados estáveis em água foi calculada por meio dos índices de agregação: diâmetro médio ponderado (D MP), módulo de finura (M F) e percentagem de agregados > 2,00 mm (AGRI) que apresentaram diferença significativa na avaliação das áreas com e sem aplicação de vinhaça. $\mathrm{N}$ as áreas com vinhaça o carbono orgânico total apresentou melhores correlações com os indicadores DMP, AGRI e MF e as maiores percentagens de agregados estáveis em água $>2,00 \mathrm{~mm}$ ocorreram na profundidade de 0-0,20 $\mathrm{m}$, nas áreas com vinhaça.

Palavras-chave: índice de agregação do solo, carbono orgânico total, qualidade de solo, Saccharum

\section{Soil attributes and soil aggregate stability relationship in sugarcane fields with and without vinasse application}

\begin{abstract}
A B ST RACT
The present study had as objective of evaluating of the effects of vinasse application on aggregate stability and in indice of soil aggregation as well as dependence of physical properties of the soil in sugarcane growing areas in south coast of Pernambuco, Brazil. Soil samples were collected from 0-0.20 and 0.20-0.40 m layers in 12 areas with and without vinasse application. Analysis was carried out to characterize soil and to evaluate, water dispersed clay, degree of clay flocculation, total organic carbon and organic matter in soil. The stability of aggregates in water was calculated through the aggregation indice: weighted mean diameter (PMD), module of fineness (MF) and percentage of aggregate $>2.00$ $\mathrm{mm}$ (AGRI) which showed significant differences between areas with and without vinasse. In areas with vinasse the organic carbon presented better correlations with PMD, MF and AGRI than without vinasse. The higher percentage of AGRI occurred in $0-0.20 \mathrm{~m}$ in areas with application of vinasse.
\end{abstract}

Key words: aggregation indices, organic matter, soil quality, Saccharum 


\section{INTRODUÇÃO}

As propriedades do solo podem ser utilizadas como parâmetros para avaliar a relação entre o manejo e a qualidade do solo (Lima et al., 2007). Desta forma, o manejo provoca alterações nas propriedades do solo que podem não suportar, enfim, a prática agrícola (Silva et al., 2005b). Além disso, o uso e o tempo de utilização são fatores que também promovem alterações nas propriedades do solo. Dentre essas propriedades pode-se citar a agregação das partículas, que está diretamente ligada com a estrutura do solo (Loss et al., 2009; Coutinho et al., 2010).

O processo de agregação envolve um conjunto de elementos como argila, ferro, alumínio e matéria orgânica, que atuam como agentes cimentantes unindo as partículas do solo, o que, de acordo com Corrêa et al. (2009) aumenta sua agregação. Os agregados estáveis em água restabelecem a porosidade do solo influenciando no processo de infiltração e a resistência à erosão. Entretanto, os agregados não estáveis podem desaparecer ao mínimo impacto provocado pelas gotas de chuva. Por sua vez, a estabilidade dos agregados pode ser definida como uma força resistente a uma ação mecânica passível de degradar a estrutura do solo tal como, também, a capacidade do solo de resistir às forças compactantes (Silva et al., 2006).

A análise de determinados atributos, como a argila dispersa em água (ADA), que reflete diretamente sobre a formação da compactação nos solos e também o grau de floculação das Argilas (GFA) que influencia na agregação das partículas do solo, são importantes para os estudos de conservação dos solos (Santos et al., 2010).

Desta forma, ao se fazer uso de sistemas de cultivo que aumentem o teor de matéria orgânica do solo, haverá contribuição para o aumento da estabilidade de agregados e, consequentemente, para a melhoria da qualidade física do solo (Vasconcelos et al., 2010). A estabilização de agregados pode ser avaliada por parâmetros de agregação que poderão dar suporte às práticas agrícolas adotadas.

Estudos que exploram as relações entre os agregados e a matéria orgânica (MO) vêm sendo realizados nos últimos anos, como os que identificaram, além da fração mineral, a fauna do solo, raízes e variáveis ambientais, como fatores que favorecem a formação dos agregados do solo (Salton et al., 2008). Segundo Bastos et al. (2005) a aplicação de compostos orgânicos melhorou a estabilidade em água dos agregados. Demarchi et al. (2011) encontraram, ao realizar estudo sobre a estabilidade dos agregados do solo em cinco sistemas de uso e ocupação, melhores resultados para as áreas com pastagens e mata nativa. Para Vasconcelos et al. (2010) diferentes sistemas de manejo promoveram alterações na distribuição de agregados enquanto a estabilização dos agregados na camada superficial dependeu do teor de matéria orgânica e da ação dos ciclos sucessivos de secagem e umedecimento do solo.

As modificações que ocorrem nas estruturas dos solos agrícolas em razão do uso e do manejo inadequados são sumamente importantes para a escolha de sistemas que priorizem a conservação das propriedades dos solos e aumentem o potencial produtor dessas áreas (Rozane et al.,
2010). Assim, quando exposto a cultivos intensivos, a sistemas de manejo inadequados e ao uso de implementos agrícolas pesados, o solo pode perder ou ter sua estrutura original alterada contribuindo para a formação de camadas compactadas que provocam redução no volume dos poros e aumentam a densidade do solo causando mudanças na estabilidade dos agregados do solo (Maria et al., 2007).

Objetivou-se, então, avaliar os efeitos da aplicação da vinhaça na estabilidade dos agregados do solo e correlacionar os índices de agregação com atributos físicos e químicos do solo em áreas cultivadas com cana-de-açúcar no litoral sul do estado de Pernambuco.

\section{Material e MÉTOdos}

O estudo foi realizado no litoral sul do estado de Pernambuco, em doze áreas cultivadas com cana-de-açúcar (Saccharum officinarum) pertencentes às Usinas Estreliana (Áreas 1,2 e 10 - Usina A), União \& Indústria (Áreas 3, 4, 5 e 11 - Usina B) e Trapiche (Áreas 6,7, 8, 9 e 12 - Usina C) nos municípios de Ribeirão, Primavera e Sirinhaem, respectivamente.

As coordenadas geográficas das primeira e última áreas, são: Área 1: $8^{\circ} 52^{\prime} 4,06^{\prime \prime} \mathrm{Se} 35^{\circ} 38^{\prime} 48,47^{\prime \prime} \mathrm{W}$. Área 12: $8^{\circ} 56^{\prime} 22$, $65^{\prime \prime} \mathrm{S} \mathrm{e} 35^{\circ} 51^{\prime} 25,45^{\prime \prime} \mathrm{W}$. O clima da região é tropical úmido do tipo As' ou pseudotropical, de acordo com a classificação climática de Köppen e se caracteriza por ser quente e úmido, com chuvas de outono a inverno, com temperaturas médias anuais variando em torno de $24^{\circ} \mathrm{C}$.

Nas Áreas avaliadas foram eleitas duas situações de manejo do solo: 6 áreas irrigadas com vinhaça e diferentes históricos de aplicação e 6 áreas sem vinhaça (Tabela 1). Em média, a área com vinhaça vem sendo fertirrigada há 4 anos.

As amostras foram coletadas em quatro pontos espaçados $50 \mathrm{~m}$, formando um quadrado dentro do talhão, entre os meses de janeiro e fevereiro de 2011. Os pontos de amostragem foram localizados nas entrelinhas da cultura da cana-de-açúcar, nos quais trincheiras foram abertas para a retirada das amostras nas camadas de 0-0,20 e 0,20-0,40 m resultando no total de 48 pontos e 96 amostras de solo.

Para as camadas estabelecidas coletou-se, em cada ponto, uma amostra de estrutura deformada, com auxílio de enxadeco e uma com estrutura indeformada, através de auxílio de um trado de diâmetro $4,89 \mathrm{~cm}$, altura $2,63 \mathrm{~cm}$ e volume $49,37 \mathrm{~cm}^{3}$. Foram retirados, aproximadamente, $600 \mathrm{~cm}^{3}$ de solo por camada, acondicionados em sacos plásticos transparentes, identificados e conduzidos ao Laboratório de Mecânica do Solo e Aproveitamento de Resíduos da Universidade Federal Rural de Pernambuco (UFRPE); em seguida foram armazenados para posteriores análises físicas e de carbono orgânico total (COT).

Na Tabela 2 tem-se a caracterização das áreas estudadas.

Parte das amostras foi colocada para secar ao ar (TFSA) por $72 \mathrm{~h} \mathrm{e}$, posteriormente, destorroada e passada na peneira de $2,00 \mathrm{~mm}$. A análise granulométrica foi realizada pelo método do densímetro e a densidade de partículas pelo método do balão volumétrico (EMBRAPA, 1997). 
Tabela 1. H istórico das áreas cultivadas com cana-deaçúcar na zona da mata sul do estado de Pernambuco

\begin{tabular}{|c|c|c|c|c|c|c|}
\hline Área & Aplicação & $\begin{array}{c}\text { Histórico } \\
\text { da } \\
\text { aplicação }\end{array}$ & $\begin{array}{c}\text { Adubação } \\
\text { mineral }\end{array}$ & $\begin{array}{l}\text { Prod. } \\
\text { média } \\
\left(\mathrm{t} \mathrm{ha}^{-1}\right)\end{array}$ & $\begin{array}{c}\text { Sem } \\
\text { implemento } \\
\text { agrícola (anos) }\end{array}$ & Variedade \\
\hline 1 & CV & 3 anos & CAM & 62 & 5 & SP86-4764 \\
\hline 2 & SV & - & CAM & 44 & 4 & SP86-4764 \\
\hline 3 & SV & - & CAM & 75 & 9 & SP78-4764 \\
\hline 4 & CV & 4 anos & SAM & 92 & 2 & RB92-579 \\
\hline 5 & CV & - & SAM & 58 & 2 & RB92-579 \\
\hline 6 & SV & - & CAM & 72 & 5 & RB86-3129 \\
\hline 7 & CV & 5 anos & SAM & 52 & 3 & SP81-3250 \\
\hline 8 & SV & - & CAM & 56 & 1 & SP78-4764 \\
\hline 9 & SV & - & CAM & 54 & 1 & SP78-4764 \\
\hline 10 & $\mathrm{CV}$ & 5 anos & SAM & 81 & 2 & SP81-3250 \\
\hline 11 & CV & 3 anos & SAM & 67 & 6 & SP78-4764 \\
\hline 12 & SV & - & CAM & 80 & 2 & SP93-3094 \\
\hline
\end{tabular}

CV - Com vinhaça; SV - Sem vinhaça; CAM - Com adubação mineral; SAM - Sem adubação mineral

O teor de COT foi obtido pela oxidação da matéria orgânica via úmida com dicromato de potássio em meio sulfúrico; posteriormente, foi determinada a percentagem de matéria orgânica multiplicando-se o resultado do COT por 1,724 (EMBRAPA, 1997).

Outra parcela das amostras foi utilizada para análise de estabilidade de agregados em água. Os dados referentes ao COT foram utilizados para avaliar as relações entre os índices de agregação e a MO para as análises de correlações de Pearson entre os índices de estabilidade dos agregados e as propriedades físicas do solo.

Para determinação da densidade dos solos e da umidade gravimétrica retiraram-se as amostras com o auxílio de um trado; após coletadas, as amostras foram armazenadas em cápsulas metálicas, devidamente embaladas em filme de PVC visando preservar sua umidade. Obteve-se o teor de água do solo de acordo com EMBRAPA (1997) em que as amostras foram pesadas e colocadas em estufa com temperatura de $105^{\circ} \mathrm{C}$ e novamente aferidos os pesos após atingirem constância de massa; já a porosidade total foi calculada indiretamente a partir da relação entre a densidade de partículas e a densidade dos solos.

As frações granulométricas de areia, silte e argila, foram determinadas pelo método do densímetro de Boyoucos utilizando-se um agitador mecânico como dispersante físico, durante $15 \mathrm{~min}$, para solos com textura argilosa e 5 min para solos com textura arenosa. Utilizaram-se $25 \mathrm{~mL}$ de hexametafosfato de sódio (Calgon) como dispersante químico.

A estabilidade de agregados em água foi estabelecida pelo método de Kemper \& Chepil (1965) os quais utilizaram o aparelho de oscilação vertical (Yoder, 1936), através de peneiramento em água, após pré-umedecimento lento dos agregados, por capilaridade.

Com vista à determinação da agregação do solo, $100 \mathrm{~g}$ foram umedecidos e posteriormente colocados em dois conjuntos de cinco peneiras com malhas de 2,00; 1,00;0,50;0,25 e 0,125 mm, respectivamente; em seguida, os conjuntos de peneiras foram submetidos a agitação vertical (42 ciclos por minuto) durante $15 \mathrm{~min}$, em recipiente com água.

$\mathrm{O}$ material retido em cada peneira foi colocado em recipientes para secagem em estufa a $105^{\circ} \mathrm{C}$, durante $24 \mathrm{~h}$. Os valores do diâmetro médio ponderado, DMP (Kemper \& Rosenau, 1986), do módulo de finura, MF (Freire \& Piedade Jr., 1979) e o AGRI, somatório dos porcentuais, a 2,00 mm, AGRI (Wendling et al., 2005) foram determinados segundo as fórmulas a seguir:

Tabela 2. Caracterização física das 12 áreas estudadas

\begin{tabular}{|c|c|c|c|c|c|c|c|c|c|c|}
\hline \multirow{2}{*}{ Área } & \multirow{2}{*}{ Aplicação } & \multirow{2}{*}{$\begin{array}{l}\text { Prof. } \\
\text { (m) }\end{array}$} & \multicolumn{3}{|c|}{ Granulometria $\left(\mathrm{g} \mathrm{kg}^{-1}\right)$} & \multirow{2}{*}{$\begin{array}{c}\text { DS } \\
\left(\mathrm{g} \mathrm{cm}^{3}\right)\end{array}$} & MO & $\theta$ & \multirow{2}{*}{ Classe textural } & \multirow{2}{*}{ Relevo } \\
\hline & & & Areia & Silte & Argila & & \multicolumn{2}{|c|}{$(\%)$} & & \\
\hline \multirow{2}{*}{1} & \multirow{2}{*}{ Com vinhaça } & $0-0,20$ & 514,9 & 188,1 & 297,0 & 1,38 & 2,67 & 0,20 & & Encosta \\
\hline & & $0,20-0,40$ & 504,9 & 163,1 & 332,0 & 1,50 & 1,58 & 0,20 & Franco argilo-arenosa & Encosta \\
\hline \multirow[b]{2}{*}{2} & \multirow{2}{*}{ Sem vinhaça } & $0-0,20$ & 509,9 & 198,0 & 292,0 & 1,41 & 2,61 & 0,25 & Franco argilo-arenosa & \\
\hline & & $0,20-0,40$ & 439,9 & 138,1 & 422,0 & 1,41 & 1,45 & 0,28 & Argila & varzea \\
\hline \multirow{2}{*}{3} & \multirow{2}{*}{ Sem vinhaça } & $0-0,20$ & 617,5 & 113,7 & 268,8 & 1,40 & 2,32 & 0,14 & & \\
\hline & & $0,20-0,40$ & 647,5 & 80,5 & 272,0 & 1,36 & 1,37 & 0,12 & Franco argilo-arenosa & Várzea \\
\hline \multirow{2}{*}{4} & \multirow{2}{*}{ Com vinhaça } & $0-0,20$ & 453,0 & 65,0 & 482,0 & 1,13 & 3,23 & 0,23 & & \\
\hline & & $0,20-0,40$ & 465,5 & 62,5 & 472,0 & 1,26 & 2,24 & 0,21 & Arglla & Varzea \\
\hline \multirow{2}{*}{5} & \multirow{2}{*}{ Com vinhaça } & $0-0,20$ & 628,0 & 132,5 & 239,5 & 1,55 & 2,02 & 0,13 & & \\
\hline & & $0,20-0,40$ & 613,0 & 140,0 & 247,0 & 1,63 & 1,72 & 0,11 & Franco argilo-arenosa & Encosta \\
\hline \multirow{2}{*}{6} & \multirow{2}{*}{ Sem vinhaça } & $0-0,20$ & 655,5 & 122,5 & 222,0 & 1,42 & 2,03 & 0,16 & & \\
\hline & & $0,20-0,40$ & 670,5 & 107,5 & 222,0 & 1,30 & 1,75 & 0,15 & Franco argilo-arenosa & Chä \\
\hline \multirow{2}{*}{7} & \multirow{2}{*}{ Com vinhaça } & $0-0,20$ & 492,4 & 45,3 & 462,3 & 1,39 & 2,62 & 0,25 & Argilo-arenosa & \\
\hline & & $0,20-0,40$ & 434,9 & 37,8 & 527,3 & 1,19 & 1,95 & 0,27 & Argila & Várzea \\
\hline \multirow{2}{*}{8} & \multirow{2}{*}{ Sem vinhaça } & $0-0,20$ & 664,3 & 37,8 & 300,1 & 1,37 & 2,21 & 0,20 & Franco argilo-arenosa & \\
\hline & & $0,20-0,40$ & 615,0 & 24,9 & 360,1 & 1,50 & 1,94 & 0,20 & Argilo-arenosa & ncosta \\
\hline \multirow{2}{*}{9} & \multirow{2}{*}{ Sem vinhaça } & $0-0,20$ & 556,8 & 20,6 & 422,6 & 1,52 & 2,38 & 0,20 & & \\
\hline & & $0,20-0,40$ & 490,9 & 13,7 & 497,6 & 1,46 & 2,01 & 0,23 & nosa & Várzea \\
\hline \multirow{2}{*}{10} & \multirow{2}{*}{ Com vinhaça } & $0-0,20$ & 538,4 & 47,8 & 413,8 & 1,20 & 3,32 & 0,23 & Argilo-arenosa & \\
\hline & & $0,20-0,40$ & 446,6 & 29,6 & 523,8 & 1,30 & 2,85 & 0,26 & Argila & árzea \\
\hline \multirow{2}{*}{11} & \multirow{2}{*}{ Com vinhaça } & $0-0,20$ & 703,0 & 32,8 & 264,2 & 1,72 & 2,32 & 0,16 & & \\
\hline & & $0,20-0,40$ & 673,0 & 22,8 & 304,2 & 1,69 & 1,86 & 0,15 & Franco argilo-arenosa & Varzea \\
\hline \multirow{2}{*}{12} & & $0-0,20$ & 578,0 & 55,3 & 366,7 & 1,11 & 2,02 & 0,27 & Franco argilo-arenosa & \\
\hline & Sem vinhaça & $0,20-0,40$ & 438,0 & 52,8 & 509,2 & 1,00 & 1,56 & 0,26 & Argila & Chã \\
\hline
\end{tabular}

Prof. - profundidade; DS - densidade do solo; MO - matéria orgânica do solo; $\theta$ - umidade gravimétrica 


$$
\begin{gathered}
\text { DMP }=\sum_{i=1}^{\mathrm{n}}(\mathrm{cp} \times \mathrm{p}) \\
\mathrm{MF}=\sum_{(0,50 \times 0,25)+(0,25 \times 0,125)(<0,25)] \div 100}[(4,00 \times 2,00)+(2,00 \times 1,00)+(1,00 \times 0,50)+
\end{gathered}
$$$$
\text { AGRI }=\text { wi }>2 \times 100
$$

em que:

cp - centro de cada classe de peneira

p - peso seco da amostra, $\%$

MF - módulo de finura dos agregados

$\Sigma$ - somatório da percentagem acumulada de agregados em cada classe

wi > 2 - proporção de agregados $>2,00 \mathrm{~mm}$

Os dados foram submetidos à análise de variância, as médias do DMP, MF e AGRI em relação aos sistemas de manejo e profundidades foram avaliadas pelo teste de Tukey a 5\% de probabilidade. Para analisar as relações entre os índices de estabilidade de agregados, as propriedades físicas e a matéria orgânica do solo e as relações entre os atributos do solo nas áreas estudadas, cálculos de coeficientes de correlação de Pearson foram efetuados em nível de 5 e $1 \%$ de significância utilizando-se o software estatístico SAS (SAS Institute, 2005). Para o estudo das correlações entre o DMP, o MF, o AGRI e o COT, efetuou-se o ajuste de equações em nível de 5 e $1 \%$ de significância (Origin $6.0^{\circledR}$ ).

\section{RESULTADOS E DISCUSSÃO}

Segundo a textura, os solos foram classificados como: franco-argilo-arenoso, em cinco das doze áreas (1, 3, 5, 6 e 11), argiloso na área 4 e argilo-arenoso na área 9, nas duas camadas estudadas; para as demais áreas foram encontradas duas classificações texturais diferentes para as duas camadas estudadas, franco argilo-arenoso na camada 0-0,2 m e argilosa na camada 0,2-0,4 m (2 e 12), argilo-arenoso na camada $0-0,2 \mathrm{~m}$ e argilosa na camada $0,2-0,4 \mathrm{~m}$ (7 e 10) e franco argilo-arenoso na camada 0-0,2 m e argilo-arenoso na camada 0,2-0,4 m (8) (Tabela 2).

Observou-se que os solos apresentam baixos teores de argila e elevados teores de areia (Tabela 2); apesar disto os teores de argila aumentam com a profundidade, ao contrário dos teores de areia, comportamento que pode ser atribuído principalmente à translocação de argila do horizonte A e à sua acumulação no horizonte B (Oliveira et al., 2010b) influenciando, desta forma, na distribuição das classes de agregados.

As correlações entre os índices de estabilidade de agregados (DMP, AGRI e MF), as propriedades físicas e a matéria orgânica do solo, se encontram na Tabela 3 , em que esta análise foi realizada para avaliar o grau de dependência entre os índices de estabilidade e as propriedades do solo.

Os índices se correlacionaram negativamente com a profundidade e com a densidade do solo demonstrando que, quanto maior a profundidade maior também é a densidade do solo (Tabela 3); para a matéria orgânica o maior valor foi encontrado na primeira profundidade de solo (Tabela 2). Oliveira et al. (2010a) observaram que, na avaliação dos agregados estáveis em água, os índices AGRI, IEA, MF e DMPdiminuíram com a profundidade do solo entre os manejos sem irrigação e com vinhaça devido a uma concentração menor da matéria orgânica nos horizontes inferiores.

Tabela 3. Correlações entre os índices de estabilidade de agregados, as propriedades físicas e a matéria orgânica do solo

\begin{tabular}{lccccccc}
\hline & Prof. & DS & $\boldsymbol{\theta}$ & S & ADA & MO & PT \\
AGRI & $-0,37 *$ & $-0,33^{*}$ & $0,37^{*}$ & $0,34^{*}$ & $0,21 * *$ & $0,81^{* *}$ & $0,27 *$ \\
DMP & $-0,32^{*}$ & $-0,37 *$ & $0,43^{*}$ & $0,27^{*}$ & $0,26 *$ & $0,79 * *$ & $0,32^{*}$ \\
MF & $-0,25^{* *}$ & $-0,41^{*}$ & $0,46^{*}$ & $0,16 * *$ & $-0,30 *$ & $0,73^{* *}$ & $0,37 *$ \\
\hline
\end{tabular}

Prof. - profundidade, DS - densidade do solo, $\theta$ - umidade gravimétrica, S - Silte, ADA - argila dispersa em água, MO - matéria orgânica, PT - porosidade total, AGRI - percentuais de agregados $>2 \mathrm{~mm}$, DMP - diâmetro médio ponderado e MF - módulo de finura.

Significativo a nível de $0,01\left(^{* *}\right.$ e e $0,05(*)$ de probabilidade pela análise de correlação de Pearson

Correlações positivas ocorreram entre os índices de estabilidade AGRI, DMP e MF, a umidade gravimétrica, o teor de silte, a matéria orgânica e a porosidade total; embora tenha havido significância entre essas propriedades e os índices, apenas a matéria orgânica apresentou alta correlação. Os resultados obtidos por Zalamena (2008) que realizou estudos para avaliar as características físicas do solo sob diferentes usos, em solos de textura média, mostraram correlações positivas entre a matéria orgânica e os índices de estabilidade DMG, DMPe IEA. Para Wendling et al. (2012) a matéria orgânica é um agente importante na formação e estabilização dos agregados; portanto, é natural se encontrar correlação entre a matéria orgânica e os índices.

$\mathrm{O}$ MF correlacionou-se negativamente com a argila dispersa em água, como previsto visto que uma dispersão maior das partículas provocará uma estabilidade menor de agregados. De acordo com Azevedo \& Bonumá (2004) o resultado da desagregação é a dispersão; a argila dispersa em água é a fração da argila que se dispersa naturalmente em água, devido à sua maior mobilidade.

Os resultados do DMP, MF e AGRI determinados nos sistemas de manejo com duas profundidades, são apresentados na Tabela 4. Os valores médios do DMP, MF e AGRI apresentaram média geral de $1,50 \mathrm{~mm} ; 3,17$ e $29,84 \%$, respectivamente.

$\mathrm{Na}$ avaliação das profundidades isoladamente, observa-se diferença estatística com diminuição das médias em relação à profundidade do solo. A interação vinhaça x profundidade não foi significativa para o diâmetro médio ponderado e as áreas estudadas (Tabela 4). Vasconcelos et al. (2010) encontraram diferença estatística ao avaliar a interação sistemas de manejo x profundidade em relação ao DMP, por via úmida, para as áreas com e sem vinhaça.

Em relação ao MF houve diferença estatística entre as médias da profundidade; no entanto, não ocorreu entre os manejos com e sem vinhaça. Para o AGRI foram observados resultados semelhantes aos encontrados para os índices DMP e MF mas não houve diferença para os índices de estabilidade estudados 
Tabela 4. Diâmetro médio ponderado, módulo de finura e percentagem de agregados $>2,0 \mathrm{~mm}$, determinados nos sistemas de manejo com duas profundidades

\begin{tabular}{|c|c|c|c|}
\hline \multirow{2}{*}{$\begin{array}{l}\text { Profundidade } \\
\text { (m) }\end{array}$} & \multicolumn{2}{|c|}{ Sistema de manejo } & \multirow{2}{*}{ Média } \\
\hline & Com vinhaça & Sem vinhaça & \\
\hline \multicolumn{4}{|c|}{ Diâmetro médio ponderado (mm) } \\
\hline 0,20 & 1,72 & 1,59 & $1,66 \mathrm{~A}$ \\
\hline 0,40 & 1,42 & 1,29 & $1,36 \mathrm{~B}$ \\
\hline Média & $1,57 \mathrm{a}$ & $1,44 \mathrm{a}$ & 1,50 \\
\hline \multicolumn{4}{|c|}{ Módulo de finura } \\
\hline 0,20 & 3,38 & 3,23 & $3,30 \mathrm{~A}$ \\
\hline 0,40 & 3,10 & 3,00 & $3,05 \mathrm{~B}$ \\
\hline Média & $3,24 a$ & $3,11 \mathrm{a}$ & 3,17 \\
\hline \multicolumn{4}{|c|}{ AGRI (\%) } \\
\hline 0,20 & 38,15 & 33,64 & $35,90 \mathrm{~A}$ \\
\hline 0,40 & 26,06 & 21,51 & $23,79 \mathrm{~B}$ \\
\hline Média & 32,11 a & $27,58 \mathrm{a}$ & 29,84 \\
\hline
\end{tabular}

Letras maiúsculas comparam diferenças entre as profundidades e minúsculas entre os manejos, em nível de 0,05 de probabilidade pelo teste de Tukey

em relação às áreas com e sem vinhaça nem para as diferentes áreas sob condições distintas de atributos físicos do solo (Tabela 4).

A distribuição entre os agregados estáveis em água nas classes de diâmetro para as 12 áreas estudadas nas profundidades de 0,0-0,2 e 0,2-0,4 m com e sem vinhaça, é apresentada na Figura 1.

Nas áreas com vinhaça na profundidade de 0,0-0,2 m (Figura 1A) os agregados $>2 \mathrm{~mm}$ se encontram em maior distribuição nas usinas A, B e C, com 32,58, 44,09 e 37,8\%, respectivamente, indicando a contribuição da matéria orgânica na preservação dos agregados. Oliveira et al. (2010a) obtiveram resultados semelhantes com maior distribuição nas de classes de agregados $>2 \mathrm{~mm}$ em área com vinhaça em relação aos demais manejos estudados, enquanto nas áreas sem vinhaça (Figura 1C) a maior concentração de agregados $>2,00 \mathrm{~mm}$ ocorreu na Usina C, com $32 \%$, ficando as Usinas A e B com aproximadamente $27 \%$.

$\mathrm{Na}$ profundidade de 0,2 a 0,4 a maior concentração de agregados estáveis em água $>2,00 \mathrm{~mm}$ ocorreu na Usina $\mathrm{B}$, na área com aplicação de vinhaça (Figura 1B) com aproximadamente 39,7\%; para as Usinas A e C as maiores concentrações de agregados $>2,00 \mathrm{~mm}$ ocorreram nas áreas sem vinhaça (Figura 1D) 28 e $22 \%$, respectivamente.

Nas áreas com aplicação de vinhaça houve maior concentração de agregados na classe de diâmetro entre 2,00 e 1,00 mm, na profundidade de 0,2-0,4 m, na Usina A, 22\% (Figura 1B) e para as áreas sem vinhaça a maior concentração ocorreu na Usina $C$, para as duas profundidades, 22 e $25 \%$, respectivamente (Figura $1 \mathrm{C} \mathrm{e} \mathrm{D).}$

Um dos principais agentes cimentantes das partículas do solo é a matéria orgânica. Consequentemente, é provável que parte da variação do tamanho dos agregados e dos índices de agregação, seja atribuída à variação do conteúdo de matéria orgânica no solo (Wendling et al., 2012).

Na Figura 2 são apresentadas as correlações entre o COT e os índices de agregação do solo. Na comparação entre os manejos ficou evidenciado que nas áreas em que foi aplicada a vinhaça, os indicadores de estabilidade de agregação do solo,
A.

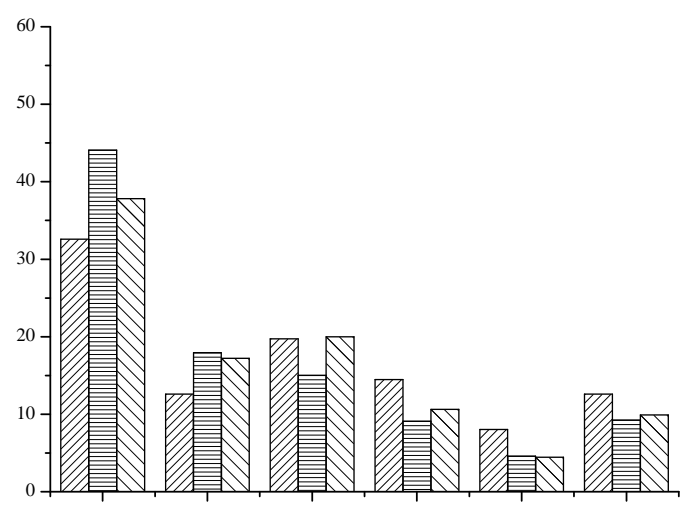

B.
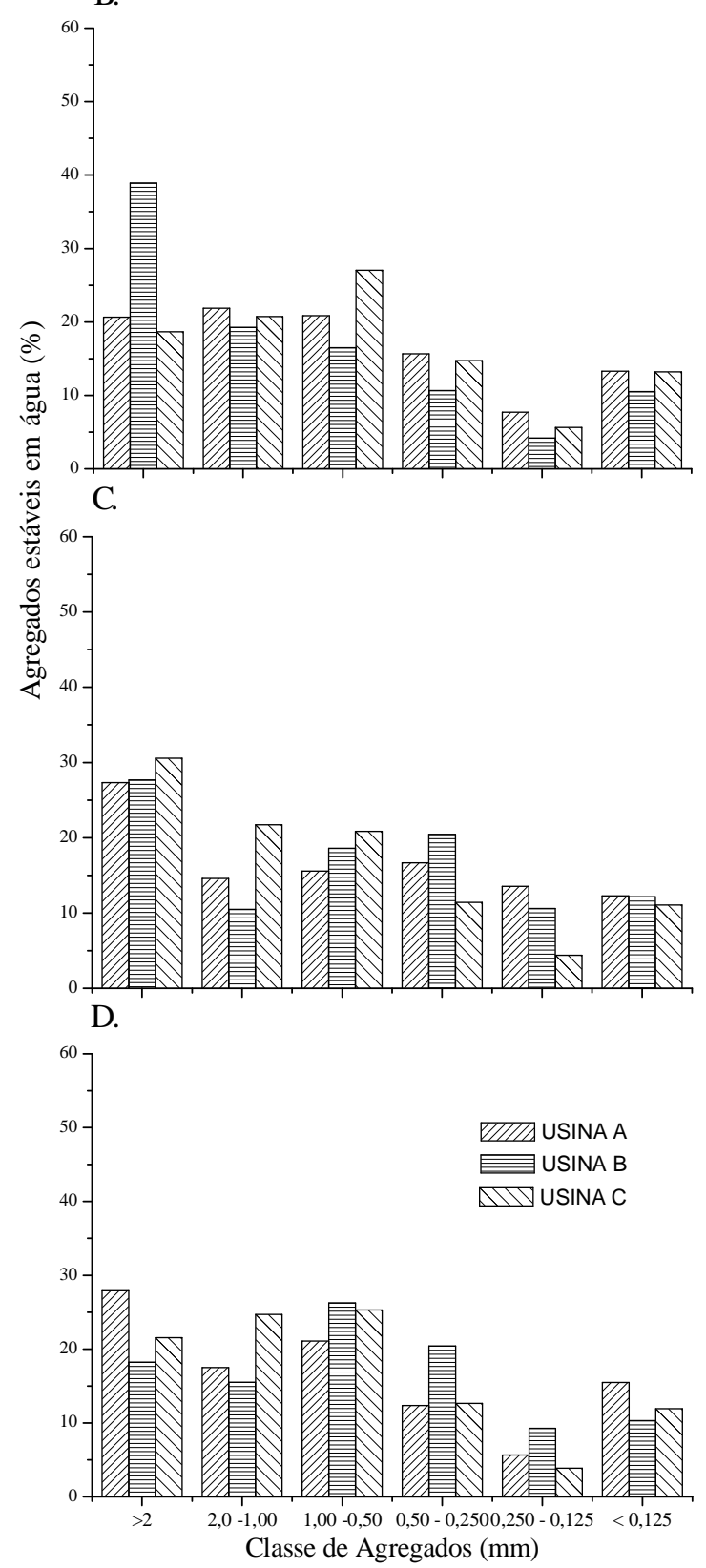

Figura 1. Distribuição dos agregados estáveis em água nas profundidades de $0-0,20$ (A) e (C) e 0,20-0,40 m (B) e (D) em áreas com vinhaça (A) e (B) e sem vinhaça (C) e (D), para três usinas localizadas no litoral sul de Pernambuco 

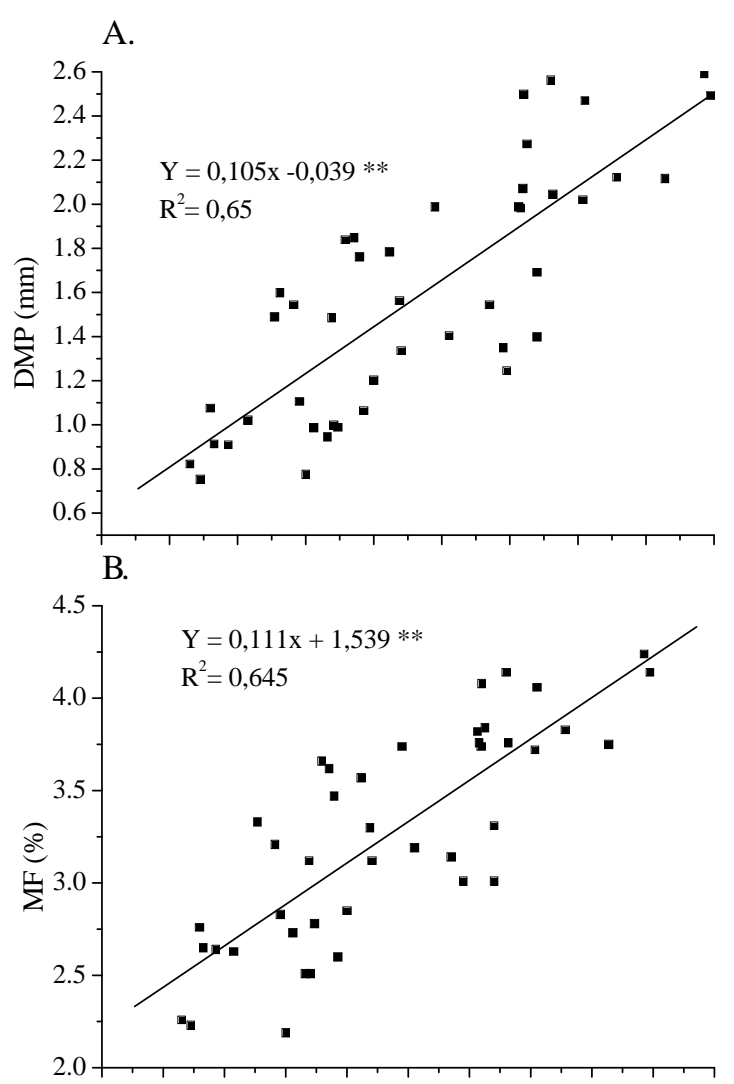

C.

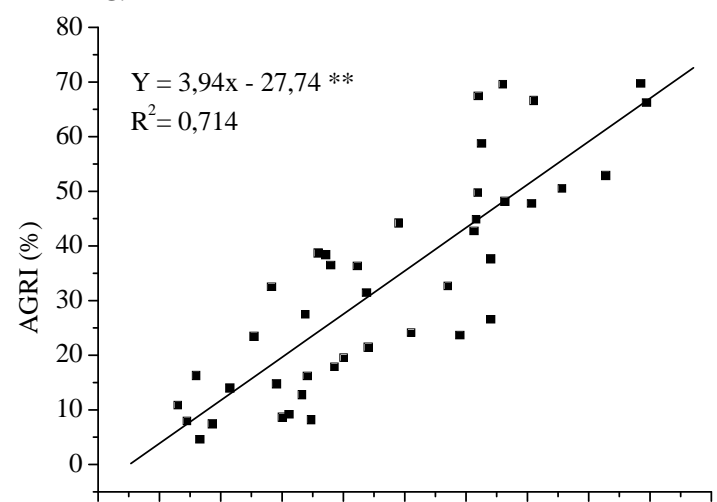

D.

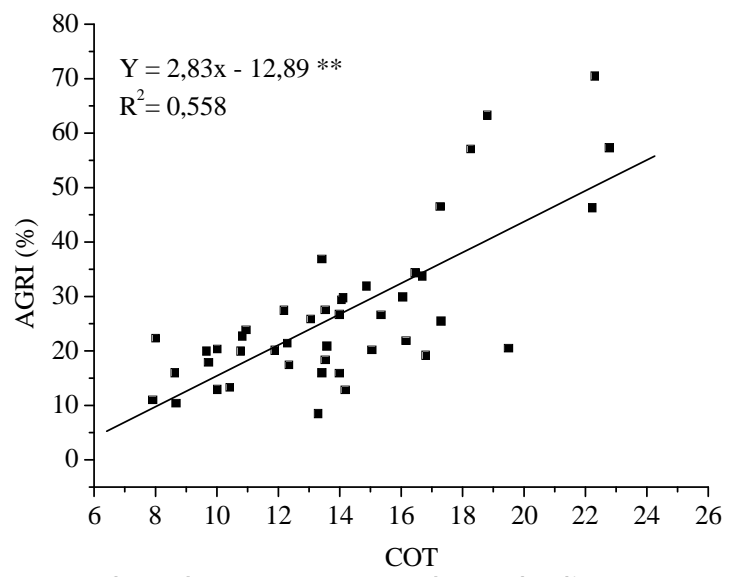

Figura 2. Correlações entre os valores do diâmetro médio ponderado (DM P), módulo de finura (M F) e percentagem de agregados $>2,00 \mathrm{~mm}$ (AG RI) com o carbono orgânico total (COT) em diferentes áreas com vinhaça $(A),(B)$ e (C), e do AG RI com COT em área sem vinhaça (D)
DMP, AGRI e MF e o COT, apresentaram fortes correlações, fato observado pelos valores do coeficiente de determinação $\left(\mathrm{R}^{2}\right)$ que são mais próximos de 1 (Figuras 2A, B e C).

O AGRI apresentou o maior coeficiente nas áreas nas quais se aplicou a vinhaça (Figura 2C e D). O DMP, por sua vez, é o índice que traduz a estabilidade dos agregados de toda a amostra (Mendes et al., 2006). Assim, os resultados obtidos indicam que a aplicação da vinhaça provoca mudanças nos agregados do solo (Figura 2A). O comportamento do DMP na área sem vinhaça é descrito pela equação a seguir, $\mathrm{y}=0,0078 \mathrm{x}$ $+0,325^{* *}$ e apresenta um $\mathrm{R}^{2}$ de 0,54 índice que também possui o menor valor do coeficiente de determinação, junto com o índice MF para as áreas com vinhaça, dentre os demais índices.

Houve correlações significativas entre os três índices avaliados no estudo e o COT, (Wendling et al., 2005; Oliveira et al., 2010a) também encontraram resultados semelhantes. Oliveira et al. (2010a) observaram $R^{2}$ altamente significativo para o DMP ao adicionar matéria orgânica ao solo. O MF foi o índice que apresentou menor coeficiente de determinação das áreas sem vinhaça, $\mathrm{R}^{2}=0,40$ com equação $\mathrm{y}=0,072 \mathrm{x}+2,078^{* *}$ que descreve este índice.

Na Tabela 5 se encontram os valores das correlações significativas entre os atributos do solo nas 12 áreas cultivadas com cana-de-açúcar, no litoral sul de Pernambuco.

Correlações negativas e positivas foram constatadas entre a profundidade e a argila dispersa em água e o grau de floculação (Tabela 5) sinalizando que esses dois atributos são inversamente proporcionais, apesar de se encontrar reduções das camadas mais superficiais nos valores de grau de floculação e, em consequência, aumento para argila dispersa em água em solos cultivados com cana-de-açúcar.

Prado \& Centurion (2001) não observaram significância nos valores entre a profundidade e a argila dispersa em água e o grau de floculação (Tabela 5) atribuindo tal feito à menor contribuição da matéria orgânica nas camadas inferiores do que nas superiores uma vez que a última é mais beneficiada com a deposição de restos vegetais ocorrendo, então, maior acúmulo de matéria orgânica.

Segundo Nunes (2003) o processo de dispersão das argilas ocorre com sucessivas calagens e adubações realizadas nos solos em período de produção. As práticas de manejo, como preparo do solo, adubações e calagens intensivas provocam, quando executadas de modo incorreto, alterações físicas do solo, refletindo nos atributos físicos, químicos, físico-químicos e atividades biológicas (Santos et al., 2010).

Quanto às correlações entre a umidade gravimétrica e os atributos físicos do solo (Tabela 5) resultados relevantes foram observados com a profundidade do solo, DS, e com as frações granulométricas de argila, areia, silte, areia grossa e areia fina. É bem provável que a umidade se tenha correlacionado positivamente com a argila, em virtude desta fração possuir maior área de superfície específica que a da areia, facilitando a interação com a umidade (Braida et al., 2006).

Os valores para a correlação entre a $\theta$ e a densidade do solo (Tabela 5) são esperados haja vista que, com seu aumento, a disponibilidade de água para o perfil do solo tende a diminuir mostrando uma provável compactação nas áreas. 
Tabela 5. Correlações entre os atributos do solo nas 12 áreas cultivadas com cana-de-açúcar, no litoral sul de Pernambuco

\begin{tabular}{|c|c|c|c|c|c|c|c|c|c|c|c|c|c|}
\hline & Prof. & $\theta$ & DS & DP & $\overline{\text { ARG }}$ & $\bar{A}$ & 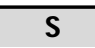 & $\overline{A G}$ & $\overline{A F}$ & $\overline{\text { ADA }}$ & $\overline{\text { GFA }}$ & MO & PT \\
\hline Prof. & & $0,13^{* *}$ & & & & & & & & $-0,26 *$ & $0,30 *$ & $-0,45^{*}$ & \\
\hline$\theta$ & & & $-0,46 *$ & $-0,31^{*}$ & $0,87^{*}$ & $-0,88^{*}$ & $0,55^{*}$ & $-0,64^{*}$ & $0,60 *$ & $0,28^{*}$ & $0,44^{*}$ & $0,23^{*}$ & $0,35^{*}$ \\
\hline DS & & $-0,46 *$ & & $0,2^{*}$ & $-0,40^{*}$ & $0,40 *$ & $-0,20 *$ & $-0,23^{*}$ & $-0,13^{* *}$ & & $-0,26 *$ & & $-0,95 *$ \\
\hline DP & & $-0,31^{*}$ & $0,20 *$ & & $-0,34^{*}$ & $0,35^{*}$ & $-0,15^{*}$ & $0,33^{*}$ & $-0,20 *$ & $-0,18 *$ & $-0,14 * *$ & $-0,27^{*}$ & \\
\hline ARG & & $0,87^{*}$ & $-0,40 *$ & $-0,34^{*}$ & & $-0,95^{*}$ & $0,36^{*}$ & $-0,66^{*}$ & $0,51^{*}$ & $0,35^{*}$ & $0,46^{*}$ & $0,19 *$ & $0,28 *$ \\
\hline A & & $-0,88^{*}$ & $0,40^{*}$ & $0,35^{*}$ & $-0,95^{*}$ & & $0,79 *$ & $0,79 *$ & $-0,65^{*}$ & $-0,44^{*}$ & $-0,37^{*}$ & $-0,23^{*}$ & $-0,28^{*}$ \\
\hline S & & $0,55^{*}$ & $-0,20 *$ & $-0,15^{*}$ & $0,36 *$ & $-0,59 *$ & & $-0,66^{*}$ & $0,80^{*}$ & $0,45^{*}$ & & $0,20 *$ & $0,13^{* *}$ \\
\hline AG & & $-0,64^{*}$ & $0,23^{*}$ & $0,33^{*}$ & $-0,66 *$ & $0,79 *$ & $-0,66 *$ & & $-0,81^{*}$ & $-0,49 *$ & $-0,12^{* *}$ & $-0,20 *$ & $-0,12 * *$ \\
\hline$A F$ & & $0,6^{*}$ & $-0,13^{* *}$ & $-0,20^{*}$ & $0,51^{*}$ & $-0,65^{*}$ & $-0,80 *$ & $-0,81^{*}$ & & $0,51^{*}$ & & & \\
\hline ADA & $-0,26 *$ & $0,28 *$ & & $-0,18^{*}$ & $0,35^{*}$ & $-0,44^{*}$ & $0,45^{*}$ & $-0,49 *$ & $0,51^{*}$ & & $-0,50^{*}$ & $0,35^{*}$ & $-0,15^{* *}$ \\
\hline GFA & $0,30 *$ & $0,44^{*}$ & $-0,26 *$ & $-0,14^{* *}$ & $0,47^{*}$ & $-0,37^{*}$ & & $-0,12^{* *}$ & & $-0,5^{*}$ & & & $0,24 *$ \\
\hline MO & $-0,45^{*}$ & $0,23 *$ & $-0,12 * *$ & $-0,27 *$ & $0,19 *$ & $-0,23^{*}$ & $0,2^{*}$ & & & $0,35 *$ & & & \\
\hline PT & & $0,35 *$ & $-0,95 *$ & & $0,28 *$ & $-0,27^{*}$ & $0,13^{* *}$ & & & $-0,15^{* *}$ & $0,24^{*}$ & & \\
\hline
\end{tabular}

Prof. - profundidade; $\theta$ - umidade gravimétrica; DS - densidade do solo; $\mathrm{CO}_{2}$ - evolução $\mathrm{C}-\mathrm{CO}_{2} ; \mathrm{DP}$ - densidade de partículas; $\mathrm{AT}$ - argila total; A - areia; $\mathrm{S}$ - silte; AG - areia grossa; AF - areia fina; ADA - argila dispersa em água; GFA - grau de floculação das argilas; MO - matéria orgânica; PT - porosidade total

Significativo a nível de $0,01\left(^{* *}\right)$ e $0,05(*)$ de probabilidade pela análise de correlação de Pearson

A DS mostrou correlações significativas com as frações granulométricas do solo e também com os atributos químicos (Tabela 5) obtendo correlações negativas com a $\theta$, além das frações de AT e S, AF, o GFA, o teor de MO e com a PT. Silva et al. (2005a) observaram, ao estudar os efeitos do sistema de preparo do solo de longa duração, 17 anos, sobre os atributos físicos relacionados ao armazenamento de água disponível de um Argissolo, que o aumento da DS acompanhou a diminuição da PT.

Dentre as frações granulométricas se destacam a ARG e a A que se correlacionaram com os atributos do solo, $\theta$, DS, DP, com a fração de S, AG, AF, ADA, GFA, MO e PT com exceção da profundidade (Prof.) (Tabela 5). Silva et al. (2005a) relataram correlação positiva entre a AT e o GFA mas não encontraram significância entre a AT e a PT. MO correlacionou-se positivamente com a umidade, as frações de ARG e S e também com a ADA mas negativamente com a prof, DP, a fração de A e AG.

\section{CONCLUSÕES}

1. O carbono orgânico total apresentou melhores correlações com os indicadores DMP, AGRI e MF, em áreas com vinhaça.

2. As maiores percentagens de agregados estáveis em água $>2,00 \mathrm{~mm}$ ocorreram na profundidade de $0,0-0,2 \mathrm{~m}$, nas áreas com vinhaça.

3. Os valores do DMP, AGRI e MF não foram influenciados pelos sistemas de manejo adotados, apenas nas profundidades das diferentes áreas estudadas.

4. O manejo com vinhaça contribuiu para a preservação dos agregados estáveis em água das áreas com média de 4 anos de aplicação.

\section{AgradeCimentos}

Ao Conselho Nacional de Desenvolvimento Científico e Tecnológico $(\mathrm{CNPq})$ e à Fundação de Amparo à Ciência e Tecnologia do Estado de Pernambuco (FACEPE), pela concessão das bolsas em prol da realização deste estudo.

\section{LITERATURA CITADA}

Azevedo, A. C.; Bonumá, A. S. Partículas coloidais, dispersão e agregação em Latossolos. Ciência Rural, v.34, p.609-617, 2004.

Bastos, R. S.; Mendonça, E. S.; Venegas, V. H. A.; Corrêa, M. M.; Costa, L. M. Formação e estabilização de agregados do solo influenciados por ciclos de umedecimento e secagem após adição de compostos orgânicos com diferentes características hidrofóbicas. Revista Brasileira de Ciência do Solo, v.29, p.21-31, 2005.

Braida, J. A.; Reichert, J. M.; Veiga, M.; Reinert, D. J. Resíduos vegetais na superfície e carbono orgânico do solo e suas relações com a densidade máxima obtida no ensaio Proctor. Revista Brasileira de Ciência do Solo, v.30, p.605-614, 2006.

Corrêa, J. C; Bull, L. T.; Crusciol, C. A. C.; Moraes, M. H. Alteração de atributos físicos em Latossolo com aplicação superficial de escória de aciaria, lama cal, lodos de esgoto e calcário. Revista Brasileira de Ciência do Solo, v.33, p.263272, 2009.

Coutinho, F. S.; Loss, A.; Pereira, M. G.; Rodrigues Júnior, D. J.; Torres, J. L. R. Estabilidade de agregados e distribuição do carbono em Latossolo sob sistema plantio direto em Uberaba, Minas Gerais. Comunicata Scientiae, v.1, p.100-105, 2010.

Demarchi, J. C.; Perusi, M. C.; Piroli, E. L. Análise da estabilidade de agregados de solos da microbacia do Ribeirão São Domingos, Santa Cruz do Rio Pardo - SP, sob diferentes tipos de uso e ocupação. Revista Brasileira de Tecnologia Aplicada nas Ciências Agrárias, v.4, p.07-29, 2011.

EMBRAPA - Empresa Brasileira de Pesquisa Agropecuária. Centro Nacional de Pesquisa de Solos. Manual de métodos de análise de solo. Rio de Janeiro: Embrapa Solos, 1997. 2.ed. 212p.

Freire, W. J.; Piedade Júnior, C. O módulo de finura dos agregados do solo como índice de estabilidade estrutural. Engenharia Agrícola, v.3, p.29-36, 1979.

Kemper, W. D.; Chepil, W. S. Size distribution of aggregates. In: Black,C. A.; Evans, D. D.; White, J. L.; Ensminger, L. E.; Clark, F. E. (ed.). Methods of soil analysis: Physical and mineralogical properties, including statistics of measurement and sampling. Part 1. Madison: American Society of Agronomy, 1965. 2.ed. p.499-510. 
Kemper, W. D.; Rosenau, R. C. Aggregate stability and size distribution. In: Klute, A. (ed.). Methods of soil analysis. Part 1. Madison: American Society of Agronomy, 1986. p.425-442.

Lima, H. V.; Oliveira, T. S.; Oliveira, M. M.; Mendonça, E. S.; Ferreira Lima, P. J. B. Indicadores de qualidade do solo em sistemas de cultivo orgânico e convencional no semi-árido cearense. Revista Brasileira de Ciência do Solo, v.31, p.10851098, 2007.

Loss, A.; Pereira, M. G.; Schultz, N.; Anjos, L. H. C.; Silva, E. M. R. Atributos químicos e físicos de um Argissolo Vermelhoamarelo em sistema integrado de produção agroecológica. Pesquisa Agropecuária Brasileira, v.44, p.68-75, 2009.

Maria, I. C.; Kocssi, M. A.; Dechen, S. C. F. Agregação do solo em área que recebeu lodo de esgoto. Bragantia, v.66, p.291298, 2007.

Mendes, F. G.; Melloni, E. G. P.; Melloni, R. Aplicação de atributos físicos do solo no estudo da qualidade de áreas impactadas, em Itajubá/MG. Cerne, v.12, p.211-220, 2006.

Nunes, L. A. P. L. Qualidade de um solo cultivado com café e sob mata secundária no município de Viçosa-MG. Viçosa: UFV, 2003. 102p. Tese Doutorado

Oliveira, V. S.; Rolim, M. M.; Vasconcelos, R. F. B.; Pedrosa, E. M. R. Distribuição de Agregados e carbono orgânico em um Argissolo Amarelo distrocoeso em diferentes manejos. Revista Brasileira de Engenharia Agrícola e Ambiental, v.14, p.907-913, 2010a.

Oliveira, V. S.; Rolim, M. M.; Vasconcelos, R. F. B.; Pedrosa, E. M. R. Compactação de um Argissolo Amarelo distrocoeso submetido a diferentes manejos. Revista Brasileira de Engenharia Agrícola e Ambiental, v.14, p.914-920, 2010 b.

Prado, R. M.; Centurion, J. F. Alterações na cor e no grau de floculação de um Latossolo Vermelho-escuro sob cultivo contínuo de cana-de-açúcar. Pesquisa Agropecuária Brasileira, v.36, p.197-203, 2001.

Rozane, D. E.; Centurion, J. F.; Romualdo, L. M.; Taniguchi, C. A. K.; Trabuco, M.; Alves, A. U. Estoque de carbono e estabilidade de agregados de um Latossolo Vermelho distrófico, sob diferentes manejos. Bioscience Journal, v.26, p.24-32, 2010.

Salton, J. C.; Mielniczuk, J.; Bayer, C.; Boeni, M.; Conceição, P. C.; Fabrício, A. C.; Macedo, M. C. M.; Broch, D. L. Agregação e estabilidade de agregados do solo em sistemas agropecuários em Mato Grosso do Sul. Revista Brasileira de Ciência do Solo, v.32, p.11-21, 2008.
Santos, L. N. S.; Passos, R. R.; Silva, L. V. M.; Oliveira, P. P.; Garcia, G. O.; Cecílio, R. A. Avaliação de alguns atributos físicos de um Latossolo Vermelho-amarelo sob diferentes coberturas vegetais. Bioscience Journal, v.26, p.940-947, 2010.

SAS - Institute Inc. SAS/STAT 9.0 User's guide. Cary: SAS Institute Inc. 2005.

Silva, A. J. N.; Cabeda, M. S. V.; Carvalho, F. G. Matéria orgânica e propriedades físicas de um Argissolo Amarelo coeso sob sistemas de manejo com cana-de-açúcar. Revista Brasileira de Engenharia Agrícola e Ambiental, v.10, p.579$585,2006$.

Silva, M. A. S.; Mafra, A. L.; Albuquerque, J. A.; Bayer, C.; Mielniczuk, J. Atributos físicos do solo relacionados ao armazenamento de água em um Argissolo Vermelho sob diferentes sistemas de preparo. Ciência Rural, v.35, p.544$552,2005 \mathrm{a}$.

Silva, R. R.; Silva, M. L. N.; Ferreira, M. M. Atributos físicos indicadores da qualidade do solo sob Sistemas de manejo na bacia do alto do Rio Grande - MG. Ciência Agrotécnica, Lavras, v.29, p.719-730, 2005b.

Vasconcelos, R. F. B.; Cantalice, J. R. B.; Oliveira, V. S.; Costa, Y. D. J.; Cavalcante, D. M. Estabilidade de agregados de um Latossolo Amarelo distrocoeso de tabuleiro costeiro sob diferentes aportes de resíduos orgânicos da cana-deaçúcar. Revista Brasileira de Ciência do Solo, v.34, p.309316, 2010.

Wendling, B.; Freitas, I. C. V.; Oliveira, R. C.; Babata, M. M.; Borges, E. N. Densidade, agregação e porosidade do solo em áreas de Conversão do cerrado em floresta de pinus, pastagem e Plantio direto. Bioscience Journal, v.28, p.256$265,2012$.

Wendling, B.; Jucksch, I.; Mendonça, E. S.; Neves, J. C. L. Carbono orgânico e estabilidade de agregados de um Latossolo Vermelho sob diferentes manejos. Pesquisa Agropecuária Brasileira, v.40, p.487-494, 2005.

Yoder, R. E. A direct method of aggregate analysis of soil and a study of the physical nature of erosion losses. Journal of the American Society of Agronomy, v.28, p.337-351, 1936.

Zalamena, J. Impacto do uso da terra nos atributos químicos e físicos de solos do rebordo do Planalto - RS. Santa Maria: UFSM. 2008, 79p. Dissertação Mestrado 